\title{
A Mobile Landing Platform for Miniature Vertical Take-Off and Landing Vehicles
}

\author{
K. Dalamagkidis, S. Ioannou, K. Valavanis, E. Stefanakos
}

\begin{abstract}
Miniature Vertical Take-Off and Landing (VTOL) vehicles have limited range of operations mainly due to their limited payload capacities and power availability. To increase this range, a modified unmanned ground vehicle (UGV) is used to transport the VTOL to its target area serving as an onsite take-off/landing and possibly refueling base. A gimbaled landing platform design is proposed and the necessary equations are derived to level the platform despite of the pose (roll, pitch, yaw) of the UGV. To increase UGV endurance, a solar array is used and solar tracking capabilities for performance maximization are examined. Simulations are carried out to check the validity of the design. The case of an ATRV-Jr as the UGV base and a Raptor 90SE as the VTOL is investigated, but the design is generic enough and suitable for other UGV/VTOL vehicles.
\end{abstract}

Index Terms-ATRV-Jr, landing platform, gimbal, photovoltaic, solar, UGV, VTOL

\section{INTRODUCTION}

$\mathbf{T}$ HERE is an increasing need for more autonomy on the deployment of unmanned vehicles especially in security, surveillance and military applications. The use of unmanned aerial vehicles in the aforementioned areas, provides several advantages due to their ability to cover a large area from above or to reach points that are not easily approachable by conventional ground vehicles. However there are severe limitations to the use of UAVs, due to their low payload capabilities, small battery capacities, fuel needs and lack of landing options in most of these applications [1].

As a UGV, the ATRV-Jr is small and can be reconfigured to provide a transport base and take-off/landing facility for a miniature VTOL. Installation of a landing platform on top of the UGV is proposed, designed in such a way to allow the VTOL to take-off and land regardless of the current pose of the ATRV-Jr, while securing it during transportation. This is achieved by providing the platform with the capability of rotation around two axes perpendicular to each other. This design is not limited to the ATRV-Jr, but instead can be transferred to other UGVs with adequate payload capacities. Using this approach the range of operations for a UAV is significantly increased by taking advantage of the larger payloads of UGVs and their higher endurance. In addition

This work was supported by the US Army Research Office, Grant Number W91-11NF-06-1-0069

K. Dalamagkidis and K. Valavanis are with Unmanned Vehicle Systems, Computer Science and Engineering Department, University of South Florida, 4202 East Fowler Avenue, ENB 118, Tampa, FL 33620 (email: kvalavan@csee.usf.edu)

S. Ioannou and E. Stefanakos are with the Clean Energy Research Center, Electrical Engineering Department, University of South Florida, 4202 East Fowler Avenue, ENB 118, Tampa, FL 33620 (email: cerceeng.usf. edu) to that, the UAV may be recharged on site, thus allowing for multiple missions.

The idea of landing a VTOL vehicle on a mobile platform is not new. Storvik in [2] investigates the problem of autonomously landing a full size helicopter on a ship. This procedure is dangerous and difficult even for manned helicopters with experienced crews. Nevertheless this topic has not been investigated significantly for miniature VTOL vehicles. In fact literature research revealed only one previous approach on this subject with similar goals. A design for autonomous launching, retrieval and refueling of UAVs was developed by SPAWAR Systems Center and Allied Aerospace as part of the Autonomous UAV Mission System (AUMS) project [3]. An initial demonstration of the capabilities of this system was done in 2003. The UAV was tele-operated but in the future the landing approach will be autonomous, based on GPS and possibly augmented by a vision system developed by CMU and JPL. This system was specifically designed to be used with Allied Aerospace's iSTAR UAV and SSC San Diego's MDARS UGV and in contrast to the design presented in this paper, adaptation to other UAV designs is not straightforward.

The goal of this work is to demonstrate the feasibility of a mobile landing platform design mounted on top of a UGV and to provide an insight on how it will operate. A study of the autonomous VTOL take off and landing procedure as well as the possible UGV-VTOL interactions and possible applications is beyond the scope of this paper.

The rest of the paper is organized as follows. At first the characteristics of the UGV and VTOL investigated are presented, followed by a presentation of the gimbaled design on which the landing platform is based. In Section IV the platform rotation is modeled and the necessary equations to calculate the angles of the gimbal axes such that it is horizontal, are derived. This section is followed by a presentation of simulation results on the landing platform. The matter of UGV endurance is addressed in Section VI where a proposal is made to install a solar array on the landing platform and the advantages of that approach are presented. The paper concludes with considerations about the proposed design.

\section{ATRV-JR AND RAPTOR 90SE SPECIFICATIONS}

The ATRV-Jr is manufactured by the iRobot Corporation and is mainly used in universities and other research environments. It has the following characteristics [4]:

- Speed $(\mathrm{m} / \mathrm{s}): 1$

- Height (cm/in): 51/20 
- Length (cm/in): 78/30.7

- Width (cm/in): $63 / 24.8$

- Weight (kg/lbs): 50/110

- Payload (kg/lbs): 25/55

- Endurance: 3-5 hr

The Thunder Tiger Raptor 90SE is a middle-sized, remotecontrolled, miniature helicopter. It has been modified to include a lightweight vision system and is currently employed in monitoring and traffic surveillance applications. Its characteristics [1] are:

- Dry weight (kg/lbs): 5.8/12.8

- Payload (kg/lbs): 4/8.8

- Endurance: $18 \mathrm{~min}$

- Skid footprint (cm/in): 50x50/19.7x19.7

It is obvious that the limited endurance of the VTOL has a severe effect on the range of its applications. By taking advantage of the payload capabilities and the larger endurance of the ATRV-Jr it is possible to transport the VTOL to its target location and use its entire endurance over target, rather than spending it en route to target.

\section{LANDING PlatForm DESIGN}

The chosen design is that of a gimbaled landing platform. This design has been used extensively, albeit in a relatively smaller scale. The gimbal usually consists of 2 or 3 concentric rings that are connected with each other by axes, each of which is driven by an individual motor [5]. As a result each ring can rotate independently of the other, keeping the inner gimbaled platform horizontal and free from vibrations. This is usually achieved with the installation of gyros, which calculate the angles of rotation of the platform, thus providing the necessary information to the motors to counter any movement of the gimbal support [6]. Its main application in the past was to keep compasses in ships horizontal and to keep accelerometers horizontal in inertial navigation systems, but currently it is more commonly used to stabilize cameras on helicopters.

Gimbaled platforms allow for 1, 2 or 3-axes rotation, but a 2-axes gimbaled system is sufficient for the landing platform purposes. Also there is no need to install gyros, since the attitude data can be supplied by the IMU of the UGV.

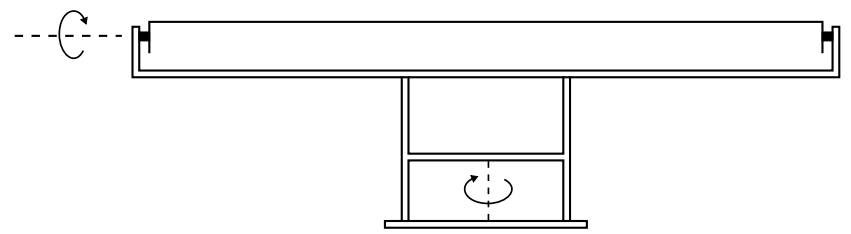

Fig. 1. Cross-section of a 2-axis gimbaled platform design.

In order for the VTOL to safely perform an autonomous vision-based landing, significant margins of error need to be accommodated and therefore the actual area of the platform is chosen to be $1 \mathrm{~m}^{2}$, which is 4 times that of the footprint of the Raptor 90SE. The platform is installed approximatelly $35 \mathrm{~cm}$ above the UGV and it can accommodate inclinations of at least $40^{\circ}$. More than that is not required because the shifting of the center of gravity of the vehicle does not allow it to move in such steep slopes. The crossection of the platform can be seen in Fig. 1 and a 3D representation of the platform on top of an ATRV-Jr is depicted in Fig. 2.

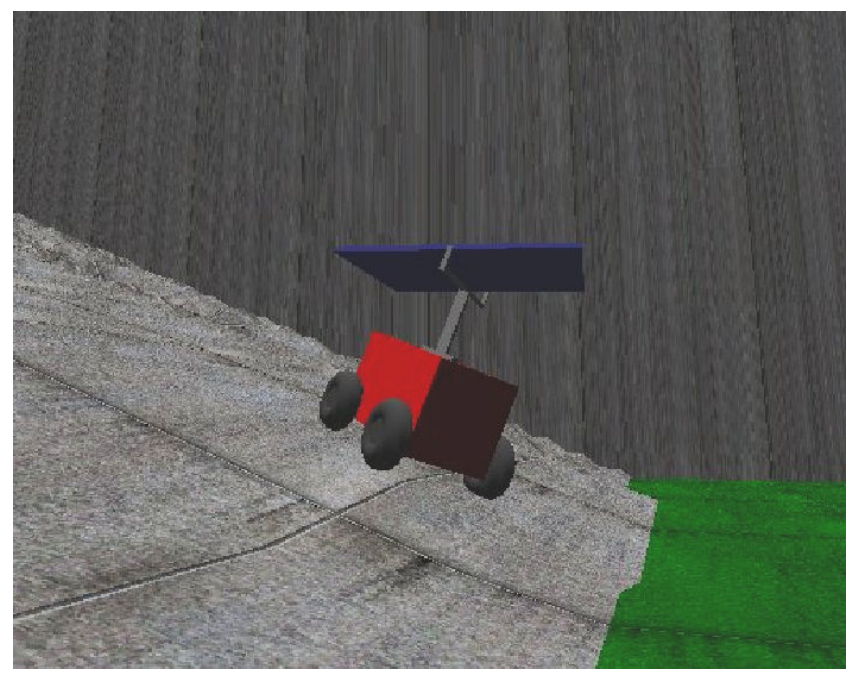

Fig. 2. 3D representation of the platform installed on top of an ATRV-Jr.

Fig. 3 shows a photo of a gimbaled platform that accommodates a small camera. It was developed during the design phase of a gyrostabilized camera, to be installed on helicopters for surveillance applications [7]. This platform uses two direct drive motors, for a total of $20 \mathrm{~W}$ power consumption.

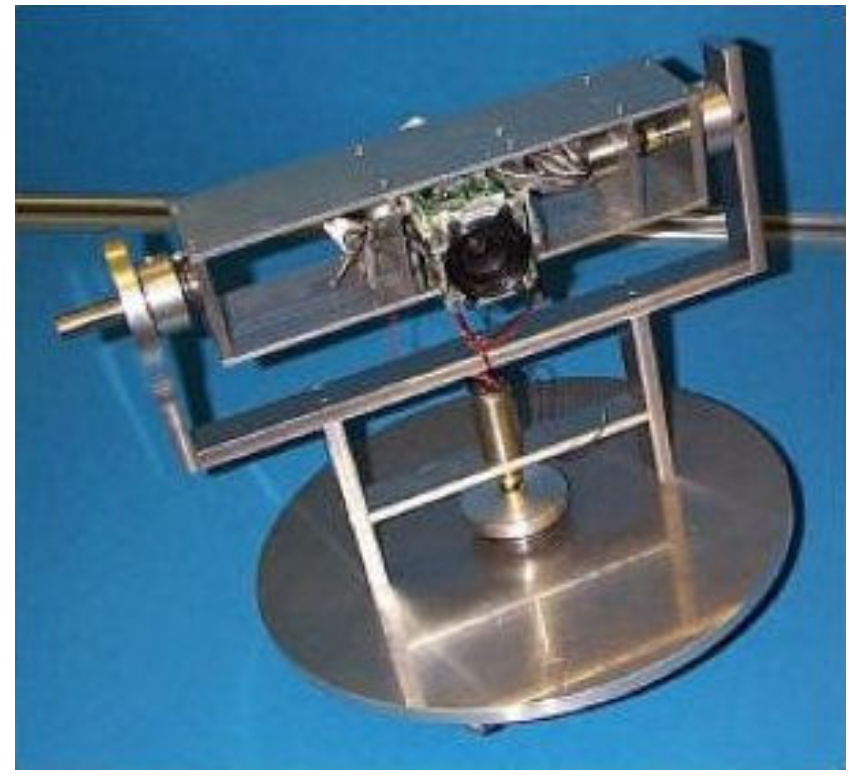

Fig. 3. Photo of a gimbaled platform used for camera stabilization. Source [7].

Since backlash is not a big issue in the landing platform design, a geared system can be alternatively used which will also take most of the load off the motors. As a consequence the platform will be more resistant to movement due to 
weight imbalance and the power requirements will remain relatively low.

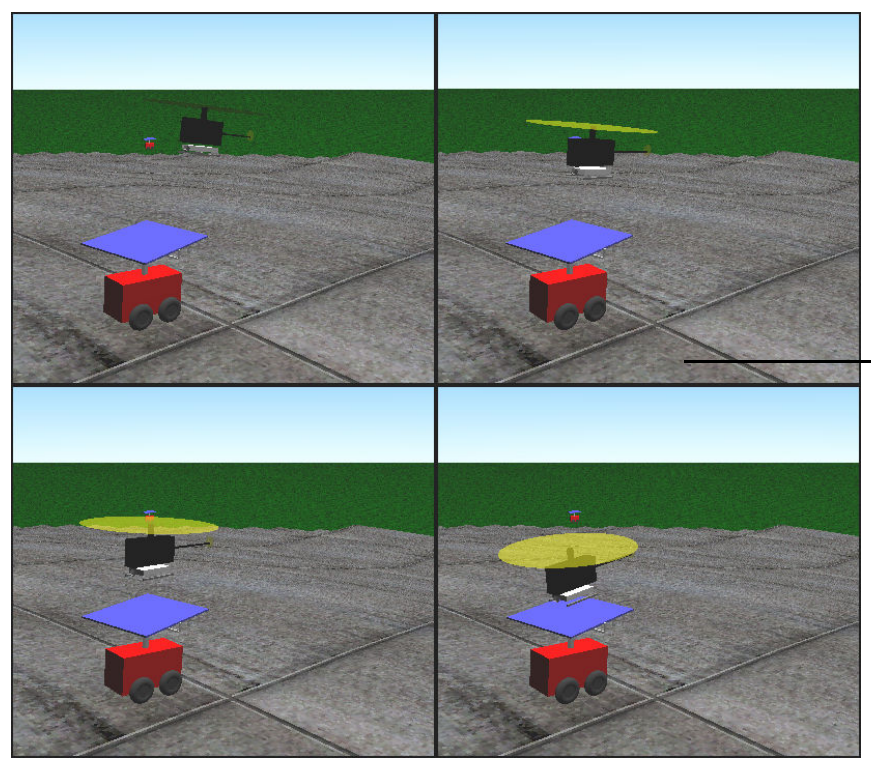

Fig. 4. Screenshots from a concept video. The modified ATRV-Jr is stopped on a slope with the platform leveled and a helicopter is on approach for landing.

\section{LEVELING THE PLATFORM}

An orthogonal Cartesian system in 3D space is used, where the $\mathrm{x}$ axis is horizontal, the $\mathrm{y}$ axis is vertical, towards the sky and the z-axis is towards the viewer (Fig. 5a). Using a matrix notation to represent the rotations of any object we have the following [8], [9]:

$$
\begin{aligned}
R(\phi) & =\left[\begin{array}{ccc}
1 & 1 & 0 \\
0 & \cos \phi & -\sin \phi \\
0 & \sin \phi & \cos \phi
\end{array}\right] \\
P(\phi) & =\left[\begin{array}{ccc}
\cos \phi & -\sin \phi & 0 \\
\sin \phi & \cos \phi & 0 \\
0 & 0 & 1
\end{array}\right] \\
Y(\phi) & =\left[\begin{array}{ccc}
\cos \phi & 0 & \sin \phi \\
0 & 1 & 0 \\
-\sin \phi & 0 & \cos \phi
\end{array}\right]
\end{aligned}
$$

where $\mathrm{R}$ is the roll matrix, $\mathrm{P}$ is the pitch matrix and $\mathrm{Y}$ is the yaw matrix. The roll, pitch and yaw angles are defined by a rotation around the $\mathrm{z}, \mathrm{x}$ and $\mathrm{y}$ axes respectively (Fig. 5b-d). Any rotated vector $\mathrm{V}$ can be calculated by a combination of the rotations mentioned above using the formula below:

$$
V^{\prime}=R\left(\phi_{1}\right) \cdot P\left(\phi_{2}\right) \cdot Y\left(\phi_{3}\right) \cdot V
$$

where the sequence with which each operation is applied is important.

Assuming that during its movement the robot reached a position where its roll, pitch and yaw angles are $\phi_{1}, \phi_{2}, \phi_{3}$,

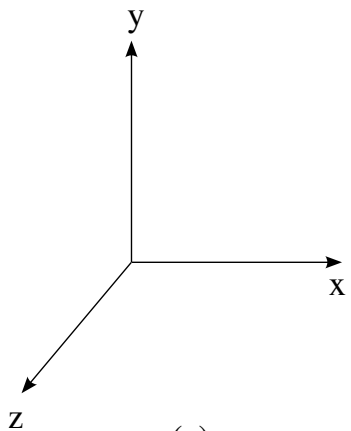

(a)

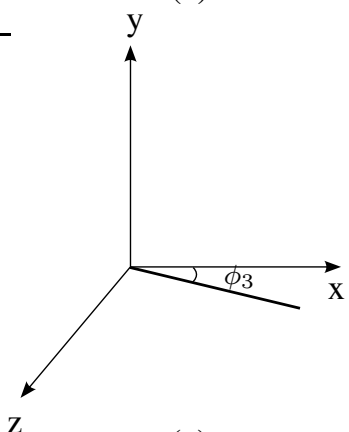

(c)

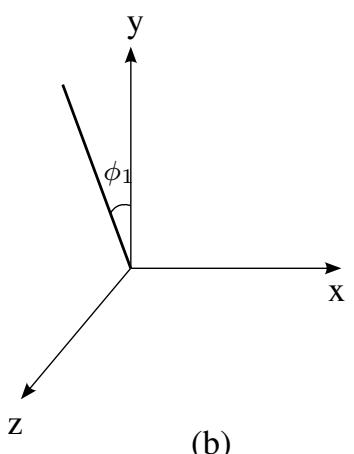

(b)

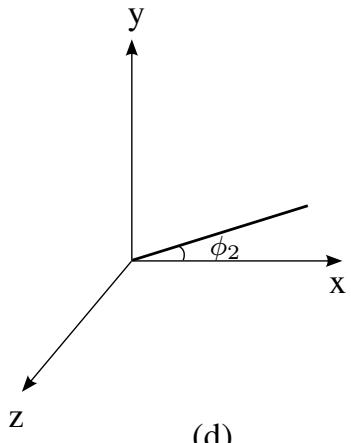

(d)
Fig. 5. (a) The Cartesian system used. (b-d) Graph b shows a vector located on the $\mathrm{y}-\mathrm{z}$ surface being rotated around the $\mathrm{x}$ axis. The angle corresponds to the roll of the vector. Similarly Graphs $\mathrm{c}$ and $\mathrm{d}$ show rotations around the $y$ and $\mathrm{z}$ axes respectively, that correspond to a change in yaw and pitch.

respectively, then it follows that as a consequence the platform as well, has the same roll, pitch and yaw with respect to the horizontal.

The landing platform is designed with the capability of rotating around two axes (Fig. 6), a vertical one and a horizontal one, which henceforth will be referred to as the heading axis and the elevation axis respectively. In order to level the platform suitable $\phi_{4}, \phi_{5}$ angles for the heading and elevation vectors respectively need to be calculated. The problem can be divided into two sub problems where the platform is first rotated around the heading axis until the elevation axis is horizontal. Then by rotating around the elevation axis until the heading axis is vertical, the platform assumes a horizontal pose.

The initial elevation vector is given by:

$$
V_{e l}=R\left(\phi_{1}\right) \cdot P\left(\phi_{2}\right) \cdot Y\left(\phi_{3}\right) \cdot V_{z}
$$

where $V_{z}$ is the z-axis unitary vector and is equal to $\left[\begin{array}{ccc}0 & 0 & 1\end{array}\right]$.

After the platform has been rotated around the heading vector, the new elevation vector becomes:

$$
V_{e l}^{\prime}=Y\left(\phi_{4}\right) \cdot V_{e l}
$$

since a change of the heading corresponds to a change of the yaw of the platform. Since the elevation axis needs to be horizontal, the dot product of the elevation vector and the $\mathrm{y}$-axis is set to zero and the $\phi_{4}$ angle is calculated. 


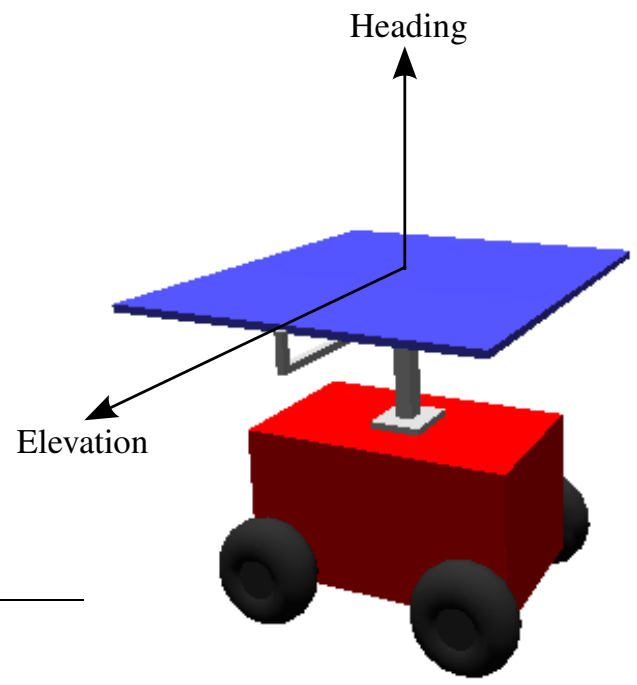

Fig. 6. The landing platform rotates around two axes, a vertical (heading) and a horizontal (elevation). These two axes are defined by the heading and elevation vectors depicted above. Using these two axes it is possible to achieve any platform orientation desired.

$$
\begin{gathered}
V_{e l}^{\prime} \bullet V_{y}=0 \Rightarrow \\
\phi_{4}=\tan ^{-1}\left(\frac{\cos \phi_{2} \cdot \sin \phi_{1}}{\sin \phi_{2}}\right)
\end{gathered}
$$

where $V_{y}$ is the $\mathrm{y}$-axis unitary vector and is equal to $\left[\begin{array}{lll}0 & 1 & 0\end{array}\right]$.

After the heading has been changed, the platform will be rotated around its new elevation vector, until the heading vector is vertical. The heading vector before this rotation is

$$
V_{h d}=R\left(\phi_{1}\right) \cdot P\left(\phi_{2}\right) \cdot Y\left(\phi_{3}\right) \cdot Y\left(\phi_{4}\right) \cdot V_{y}
$$

Since the rotation above corresponds to a change of pitch for the platform, it will become:

$$
V_{h d}^{\prime}=P\left(\phi_{5}\right) \cdot V_{h d}
$$

The angle $\phi_{5}$ is calculated so that the $V_{h d}$ vector will be vertical by setting its dot product with either the $\mathrm{x}$-axis or the $\mathrm{z}$-axis to zero.

$$
\begin{gathered}
V_{h d}^{\prime} \bullet V_{x}=0 \Rightarrow \\
\phi_{5}=\tan ^{-1}\left(\frac{\sin \phi_{2}-\tan \phi_{3} \cdot \tan \phi_{1}}{\tan \phi_{3} \cdot \sin \phi_{4}-\cos \phi_{1} \cdot \cos \phi_{2} \cdot \cos \phi_{4}}\right)
\end{gathered}
$$

where $V_{x}$ is the $\mathrm{x}$-axis unitary vector.

\section{Simulation RESUlTS}

In order to demonstrate the feasibility of the design, simulations of the robotic platform equipped with a landing platform were carried out, using the Gazebo 0.5.3(cvs121305) software [10]. This software is open source and is part of the Player/Stage/Gazebo software package. The object dynamics are calculated using the Open Dynamics Engine by
Russel Smith [11], which is also used by several simulation applications as well as games.

The landing platform was simulated as a solid mass weighing $12 \mathrm{~kg}$ and the moving understructure was modeled using several smaller geometries with a total mass of 3.2 $\mathrm{kg}$. The modeled weight of the platform corresponds to the weight of two readily available commercial solar panels, with an area equal to that of the platform and a total rated output of 120W (Sunwize SW60, [12]). The reason for this choice will become apparent in the following section.

Two motors are used to move the platform at low speeds. The elevation motor has a stop at $\pm 40^{\circ}$, while the heading motor is limited to $\pm 90^{\circ}$. The controller instructs the motors to rotate the platform until the deviation from the predetermined angle is within $\pm 1.7^{\circ}$.

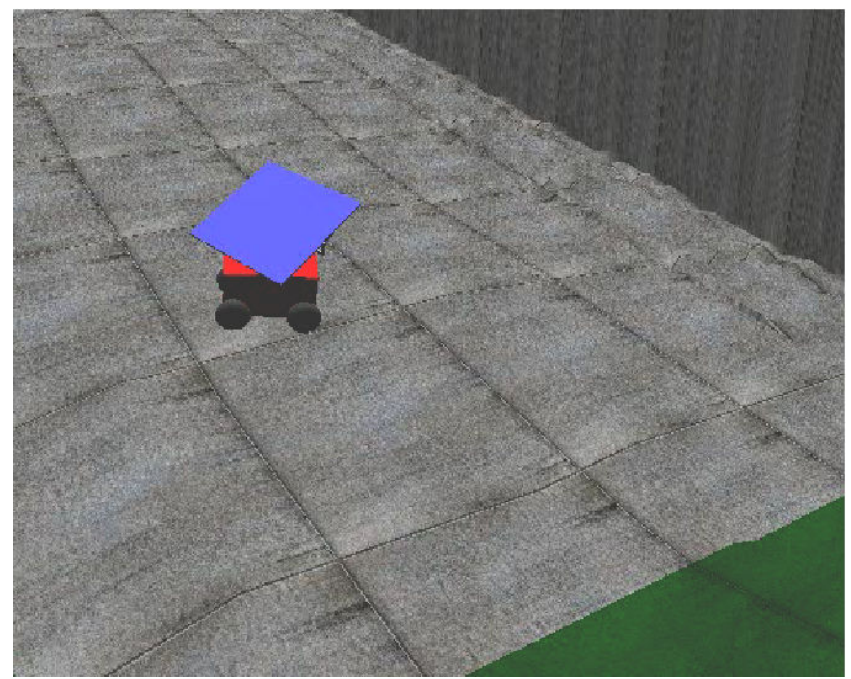

Fig. 7. The robot stopped here and is about to level the platform. The roll, pitch, yaw of the robot is $(-23.2,-3.5,65.5)$ and of the platform $(-21.4$, $33.3,104.1)$. The platform and the robot are not aligned due to a previous rotation.

In all test scenarios it was possible to rotate the platform until it was horizontal within the predetermined limits of error using only two axes rotation. Figs. 7 and 8 show a case where the platform had an initial roll and pitch of $-21.4^{\circ}$ and $33.3^{\circ}$ that was reduced to less than $1.5^{\circ}$ by a successful rotation. Further testing indicated that under the assumption of small and slow changes in the robot's pose (roll, pitch and yaw), it is possible to control the platform so that it will remain horizontal while the robot was moving, without significant increase in the error. The final actual error is determined by the backlashing effect of the geared system, as well as its speed and accuracy.

\section{USING A SOLAR ARRAY AS THE LANDING PLATFORM}

The endurance of the ATRV-Jr plays also a significant role in the range of operations of the system. A survey of its power requirements [13] is summarized in Table I. Although initially the consumption was low, after the installation of upgrades the ATRV-Jr consumes more than $400 \mathrm{~W}$ of power under full load. Nevertheless it is possible to 


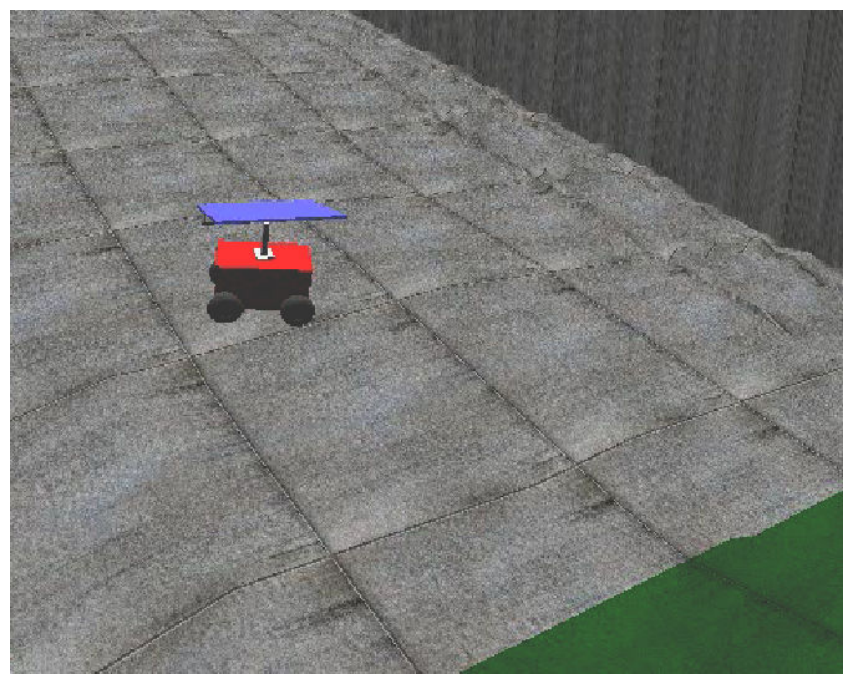

Fig. 8. The robot has succeeded in leveling the platform. The roll, pitch, yaw of the robot is still $(-23.2,-3.5,65.5)$ but that of the platform is $(-0.8$, $-1.5,-14.4)$. A tolerance of \pm 1.7 degrees deviation from zero was allowed.

TABLE I

ATRV-JR POWER REQUIREMENTS

\begin{tabular}{lccc}
\hline & \multicolumn{3}{c}{ Power Requirements } \\
& Initial & Current & Proposed [13] \\
\hline \hline $\begin{array}{l}\text { Sensors and Com- } \\
\text { puter }\end{array}$ & $40 \mathrm{~W}$ & $273 \mathrm{~W}$ & $70 \mathrm{~W}$ \\
\hline Drive motors & $131 \mathrm{~W}$ & $131 \mathrm{~W}$ & $131 \mathrm{~W}$ \\
\hline $\begin{array}{l}\text { Drive motors and } \\
\text { VTOL recharging }\end{array}$ & - & - & $25 \mathrm{~W}$ \\
\hline \hline Total & $171 \mathrm{~W}$ & $408 \mathrm{~W}$ & $226 \mathrm{~W}$ \\
\hline
\end{tabular}

lower the consumption by about $50 \%$ without compromising the quality and quantity of the sensors. In [13] it is also calculated that the ATRV-Jr needs to carry at least $2 \mathrm{KWh}$ of stored energy for a $25 \mathrm{~km} / 12$ hour mission and two VTOL recharges.

The landing platform provides a free level surface of $1 \mathrm{~m}^{2}$ that can be covered with a photovoltaic array. Although a portion of the array will be shaded by the VTOL, it is estimated that about 50 to $70 \%$ will receive solar radiation at all times. The total area available on the landing platform can be used to provide up to $120 \mathrm{~W}$ of energy under ideal conditions. Even with a more realistic performance of 60 to $70 \mathrm{~W}$, it is still possible to cover the needs of the sensors and the on-board computer. As a result while the UGV is stationary it won't consume battery power and consequently its endurance will be significantly increased, especially in regions with good weather conditions. It is estimated that $15-$ $20 \%$ of the energy demand of the mission mentioned above can be covered by such an array, thus reducing the weight and volume of on-board power storage.

Panels usually come with an aluminum frame designed to enhance their rigidity since they need to be able to support snow or ice during the winter period. We can therefore use the solar panel itself as a landing platform, without the use of any supporting surfaces. In addition to that PV panel are build to be hail resistant (BP Solar panels withstand the impact of a one inch hail stone at $52 \mathrm{mph}[14])$. Thus the array should be capable to support the weight of the VTOL and sustain the forces during landing.

Since the PV array has a very regular shape and distinct coloring, the VTOL's vision system will have no difficulty recognizing it and aligning with it. If further marking is needed the frame could be painted a different color and/or LEDs could be used to improve recognition especially in low light situations. LEDs have the advantage of high brightness, low energy consumption and long life.

When the VTOL is in the air the available rotation mechanism of the platform can be used to align the solar array with the sun, thus maximizing the former's performance. The alignment is achieved using the same methodology of the previous section. Initially the sun vector $V_{\text {sun }}$ is calculated as the normalized vector with origin the center of the platform and destination the sun.

The calculation of the $\phi_{4}, \phi_{5}$ angles for heading and elevation respectively is done like in the previous section - in two steps. First the heading vector is changed until the elevation vector $V_{e l}$ is vertical to $V_{\text {sun }}$. Then the platform is rotated around the axis defined by the elevation vector until the heading vector $V_{h d}$ is parallel to $V_{\text {sun }}$. The following two equations can be used to determine the final $\phi_{4}, \phi_{5}$ angles.

$$
\begin{gathered}
V_{\text {sun }} \bullet\left[R\left(\phi_{1}\right) \cdot P\left(\phi_{2}\right) \cdot Y\left(\phi_{3}\right) \cdot Y\left(\phi_{4}\right) \cdot V_{z}\right]=0 \\
V_{h d}=R\left(\phi_{1}\right) \cdot P\left(\phi_{2}\right) \cdot Y\left(\phi_{3}\right) \cdot Y\left(\phi_{4}\right) \cdot P\left(\phi_{5}\right) \cdot V_{y} \\
V_{\text {sun }}=\frac{V_{h d}}{\left|V_{h d}\right|}
\end{gathered}
$$

In order for the $V_{\text {sun }}$ vector to be calculated it is possible to use geographical data from the region of operations of the system in combination with chronological data (day of year and time of day). Alternatively active sun tracking methods can be also employed using a vision system or other special purpose equipment, although that would further decrease the payload capabilities of the platform.

\section{OTHER CONSIDERATIONS}

One thing that should be considered is how the VTOL can be secured on the landing platform while the UGV is moving. If the UGV is to drive up even some small inclinations, then it is quite possible that the VTOL will slip of the platform, especially if the latter is made out of glass or plastic (materials used to cover solar arrays).

A second consideration is if the VTOL is to be refueled by the UGV (either batteries, fuel or both). Recharging the batteries can be accomplished by taking advantage of the securing mechanism discussed above and using the VTOL's skids as connectors. On the other hand if refueling is also needed, the design is much more complicated and will probably call for a robotic arm that will connect to the 
VTOL regardless of the way it was landed. This robotic arm will also need a limited vision system to locate the power connection terminal on the VTOL and a low force connector so that it won't push the VTOL of the platform.

\section{CONCLUSIONS}

This paper demonstrated the feasibility of a mobile landing platform for VTOL vehicles. The two-axes gimbaled design is proven to be adequate for ensuring a horizontal landing platform for a VTOL, even during the movement of the UGV. This platform allows a significant increase in the range of operation of miniature VTOLs and as a consequence an expansion in their areas of application. Additionally the design provides the opportunity for on-site energy production from renewable energy sources, thus further increasing the VTOL's as well as the UGV's endurance.

Another advantage of the gimbaled platform is, that it can rotate around the $\mathrm{z}$-axis that passes through its center and therefore can be used also as a mounting point for a camera, allowing a full $360^{\circ}$ view. This latter feature allows tracking of moving objects even when the target object is behind the vehicle or traveling in parallel.

The problem of payload can be addressed with the use of lightweight materials for the construction of the platform. In addition to that solar panels for space applications [15] have been known to be significantly lighter and can be therefore used instead of the commercial ones. This way the impact on the total platform weight is reduced, although with a significant increase in total cost. In order to assess the feasibility of the installation of such panels, their structural characteristics need first to be investigated and the maximum impact velocity of VTOL during landing needs to be determined.

\section{REFERENCES}

[1] R. Garcia, K. Valavanis, and M. Kontitsis, "A high power, inexpensive on-board vision system for miniature unmanned vtol vehicles," CRASAR, USF, Tech. Rep. 4, 2005.

[2] M. Storvik, "Guidance system for automatic approach to a ship," Master's thesis, Norwegian University of Science and Technology, 2003.

[3] K. Mullens, E. Pacis, S. Stancliff, A. Burmeister, T. Denewiler, M. Bruch, and H. Everett, "An automated uav mission system," in AUVSI Unmanned Systems in International Security. SPAWAR Systems Center, Allied Aerospace, 2003.

[4] Space and Naval Warfare Systems Command. (2005, Sept.) Joint robotics program, tech database, robotic platforms, unmmanned ground vehicles, atrv-jr specifications. [Online]. Available: http://robot.spawar.navy.mil/images/database/ Platforms/UGV/doc/atrvjr_tech_2001.pdf

[5] Wikipedia. (2006, Jan.) Gimbal. [Online]. Available: http://en. wikipedia.org/wiki/Gimbal

[6] A. D. King, "Inertial navigation - 40 years of evolution," GEC Review, vol. 13, no. 3, 2003. [Online]. Available: http://www.marconi.com/Home/about_us/Our\%20History/ Publications\%20Archive/GEC\%20Publications\%20Archive/GEC\% 20Journals/GEC\%20Review/v13n3s/p140.pdf

[7] A. Couchman, "An investigation into the performance of a low cost rate gyroscope stabilised platform, phase 3," Master's thesis, University of Wales - Bangor, 2002.

[8] F. Dunn, 3D Math Primer for Graphics and Game Development. Wordware Publishing, Inc., 2002.

[9] M. Baker. (2005, Oct.) Euclidean space. [Online]. Available: http://www.euclideanspace.com/

[10] Sourceforge.net. (2005, Dec.) Player/stage/gazebo project page. [Online]. Available: http://playerstage.sourceforge.net/

[11] R. Smith. (2005, Oct.) Open dynamics engine. [Online]. Available: http://www.ode.org/

[12] Sunwize Technologies, Inc. (2005, Dec.) Homepage. [Online]. Available: http://www.sunwize.com/

[13] S. Ioannou, K. Dalamagkidis, K. Valavanis, E. Stefanakos, and P. Wiley, "On improving endurance of unmanned ground vehicles: The atrvjr case study," Jan. 2006, to be published.

[14] BP Solar North America. (2005, Oct.) Homepage. [Online]. Available: http://www.bp.com/modularhome.do?categoryId= 4320\& contentId $=7004540$

[15] QinetiQ Ltd. (2006, Jan.) Light weight high power solar array. [Online]. Available: http://www.qinetiq.com/home/commercial/space/ space_technology/microsat_high_power.html 